\title{
Assessment of corticospinal excitability after traumatic spinal cord injury using MEP recruitment curves: a preliminary TMS study
}

\author{
R Nardone ${ }^{1,2,3}$, Y Höller ${ }^{1,3}$, A Thomschewski ${ }^{1,3}$, AC Bathke ${ }^{4,5}$, AR Ellis ${ }^{5}$, SM Golaszewski ${ }^{1}$, \\ F Brigo ${ }^{2,6}$ and E Trinka ${ }^{1,3}$
}

Study design: Transcranial magnetic stimulation study.

Objectives: To further investigate the corticospinal excitability changes after spinal cord injury ( $\mathrm{SCl}$ ), as assessed by means of transcranial magnetic stimulation (TMS).

Setting: Merano (Italy) and Salzburg (Austria).

Methods: We studied resting motor threshold (RMT), motor evoked potential (MEP) amplitude and recruitment curve in five subjects with good recovery after traumatic incomplete cervical SCl.

Results: RMT did not differ significantly between patients and controls, whereas the slope of MEP recruitment curve was significantly increased in the patients.

Conclusion: This abnormal finding may represent an adaptive response after $\mathrm{SCl}$. The impaired ability of the motor cortex to generate proper voluntary movement may be compensated by increasing spinal excitability. The easily performed measurement of MEP recruitment curve may provide a useful additional tool to improve the assessment and monitoring of motor cortical function in subjects with SCI. Increasing our knowledge of the corticospinal excitability changes in the functional recovery after SCl may also support the development of effective therapeutic strategies.

Spinal Cord (2015) 53, 534-538; doi:10.1038/sc.2015.12; published online 10 February 2015

\section{INTRODUCTION}

After spinal cord injury (SCI), plastic changes of neural circuits occur at various level of the central nervous system. ${ }^{1}$ A number of studies have been performed to neurophysiologically characterize the functional reorganization after SCI. The impact of SCI on the brain is a complex balance between supraspinal organization and spinal recovery. ${ }^{2}$

Transcranial magnetic stimulation (TMS) represents a useful noninvasive approach for studying cortical physiology and descending motor pathways. ${ }^{3}$

Previous studies evaluating motor cortex excitability after SCI showed that the activity of intracortical inhibitory circuits may be reduced after incomplete SCI. ${ }^{4-8}$ Cortical inhibition has been studied using the application of sub-threshold TMS, ${ }^{4,6,7}$ a technique that temporarily inhibits the ongoing electromyogram (EMG), likely through the activation of intracortical inhibitory neurons with connections onto fast-conducing corticospinal axons that drive the motoneurones during voluntary contractions. ${ }^{9}$ Within several weeks of SCI, the onset of EMG suppression is $\sim 25 \mathrm{~ms}$ longer than the latency of the motor evoked potential (MEP), whereas the latency difference is only $\sim 13 \mathrm{~ms}$ in healthy controls. ${ }^{4,6}$ However, it is conceivable that a greater involvement of slow-conducting corticospinal tract axons to voluntarily activated EMG may also explain the greater delay in EMG suppression. ${ }^{4,10}$

Two single-subject reports have shown that the short-interval intracortical inhibition $(\mathrm{SICI})^{11}$ is reduced after incomplete SCI. ${ }^{5,8}$ However, SICI was examined only with one single conditioning and test stimulus intensity. Studying SICI over a range of intensities has become increasingly important for the recruitment of neurons by the test stimulus pulse that are differentially susceptible to SICI modulation, ${ }^{12-14}$ and the contribution from short-interval intracortical facilitation activated by the conditioning stimulus cannot be ruled out. ${ }^{15,16}$ It should also be considered that the stimulus intensities chosen to assess SICI are frequently based on the motor threshold, thus damage to descending motor fibers in SCI will invariably raise these TMS intensities resulting in overactivation of the intact motor cortex.

Roy et al. ${ }^{10}$ also found a reduced SICI in patients with chronic SCI; however, when the absolute stimulation intensities were matched between patients and healthy controls in terms of maximum stimulator output, both U-shaped SICI recruitment curves were produced by similar conditioning stimulation intensities.

${ }^{1}$ Department of Neurology, Christian Doppler Klinik, Paracelsus Medical University and Center for Cognitive Neuroscience, Salzburg, Austria; ${ }^{2}$ Department of Neurology, Franz Tappeiner Hospital, Merano, Italy; ${ }^{3}$ Spinal Cord Injury and Tissue Regeneration Center, Paracelsus Medical University, Salzburg, Austria; ${ }^{4}$ Department of Mathematics, Paris Lodron University, Salzburg, Austria; ${ }^{5}$ Department of Statistics, University of Kentucky, Lexington, KY, USA and ${ }^{6}$ Department of Neurological, Neuropsychological, Morphological and Movement Sciences, Section of Clinical Neurology, University of Verona, Verona, Italy

Correspondence: Dr R Nardone, Department of Neurology, Franz Tappeiner Hospital, Merano, Via Rossini 5, 39012 Merano (BZ), Italy

E-mail: raffaele.nardone@asbmeran-o.it

Received 10 September 2014; revised 30 December 2014; accepted 8 January 2015; published online 10 February 2015 
On the other hand, studies using the cortical silent period yielded contradictory results; $5,17,18$ moreover, spinal mechanisms contribute to the early phase of the cortical silent period. ${ }^{19}$

Therefore, cortical excitability after SCI needs to be further investigated, possibly using other TMS protocols or techniques.

Motor cortical excitability can also be estimated utilizing the amplitude of MEP following TMS. ${ }^{20}$ With increasing stimulus intensity, the amplitude of the MEP increases until it reaches a plateau level in healthy subjects. ${ }^{20,21}$ This increase in MEP amplitude with increasing TMS intensity is referred to as MEP recruitment curve but is also known as input/output or stimulus/response curves, as these MEP curves represent the input/output function of the motor cortex. $^{21,22}$ The sigmoid-shaped stimulus/response curve is constructed by plotting the relationship between amplitude and stimulus intensity. ${ }^{23}$ The MEP recruitment curves from paretic hand muscles in subjects with incomplete cervical SCI may provide useful information under this pathologic condition where cortical and spinal cord excitability change markedly.

We aimed at investigating whether incomplete cervical SCI affects the corticospinal excitability by assessing the MEP recruitment curve in patients with traumatic SCI and healthy controls.

\section{MATERIALS AND METHODS}

\section{Subjects}

Five subjects (mean age 44 years, range 28-54, four men and one woman, all right-handed) with chronic incomplete cervical SCI and bilateral limb involvement, classified as grades C (one patient) or D (four patients) according to the American Spinal Cord Injury Association Impairment Scale, ${ }^{24}$ were enrolled in the study. Twelve healthy volunteers (mean age 42.8, range 24-58 years, eight men and four women, all right-handed) participated as agematched controls. Clinical and demographic features of the patients are shown in Table 1.

All patients were taking antispastic medication (baclofen) and were free of any other medication known to influence the motor cortex excitability.

Patients and control subjects provided informed consent before participation in the study, which was performed according to the declaration of Helsinki and approved by the Ethics Committee. Inclusion criteria were as follows: (a) recordable MEP with an amplitude of at least $0.1 \mathrm{mV}$ in relaxed first dorsal interosseous after TMS; and (b) ability to give informed consent and comprehend instructions. Exclusion criteria were as follows: (a) severe intrinsic hand muscle weakness (Medical Research Council Scale score <3/5); (b) concomitant neurological conditions, including any history of epilepsy and polyneuropathies; (c) cognitive impairment or any substantial decrease in alertness; (d) excessive pain in any joint of the paretic extremity; (e) contraindications to TMS such as metal implants in the head; (f) advanced liver, kidney, cardiac or pulmonary disease; and (g) history of significant alcohol or drug abuse.

Table 1 Clinical and demographic characteristics of the patients with spinal cord injury

\begin{tabular}{lllcccc}
\hline Patients & $A(y)$ G & Etiology & $\begin{array}{c}\text { Time since } \\
\text { SCl }\end{array}$ & $\begin{array}{c}\text { Level/ } \\
\text { ASIA }\end{array}$ & $\begin{array}{c}\text { UL motor } \\
\text { score }\end{array}$ & $\begin{array}{c}\text { LL motor } \\
\text { score }\end{array}$ \\
\hline 1 & $54 \mathrm{M}$ & Disc prolaps & 17 & C6/D & 4138 & 4948 \\
2 & $42 \mathrm{~F}$ & Fracture & 13 & C7/D & 4242 & 4446 \\
3 & $48 \mathrm{M}$ & Fracture & 5 & C5/D & 4040 & 4444 \\
4 & $45 \mathrm{M}$ & Fracture & 11 & C6/C & 2524 & 2019 \\
5 & $28 \mathrm{M}$ & Fracture & 8 & C7/D & 4642 & 3229
\end{tabular}

Abbreviations: A, age; ASIA, American Spinal Cord Injury Association Impairment Scale; G, gender, LL, lower limb; SCl, spinal cord injury; UL, upper limb.

\section{Experimental procedures}

MEPs were recorded using pairs of $\mathrm{Ag}-\mathrm{AgCl}$ surface electrodes taped on the belly and tendon of the FDI muscles of the right side of normal controls and on the less affected side of patients. EMG signals were filtered (bandpass 5-20 $\mathrm{KHz}$ ), amplified, displayed (Dantec Keypoint, Medtronic, Skovlunde, Denmark) and stored for off-line analysis.

All subjects were asked to remain completely relaxed during TMS. They wore ear-plugs during the experiments, were seated in an armchair and supported by the arm of the chair.

TMS was performed with a high-power Magstim 200 Stimulator (Magstim Co., Whitland, Dyfed) using a figure-of-eight coil (external loop diameter $70 \mathrm{~mm}$ ) adjusted over the motor cortex at the optimum scalp position to elicit motor responses in the contralateral FDI muscle.

The resting motor threshold (RMT) was defined as the lowest stimulator output intensity capable of inducing MEPs of at least $50 \mu \mathrm{V}$ peak-to-peak amplitude in the target muscle(s) in at least half of the 10 trials. ${ }^{25}$ Both visual feedback from a PC monitor and audio feedback were used to ensure that the recorded muscle was relaxed.

We also calculated the MEP/M-wave amplitude ratio by dividing the MEP amplitude by the maximal M-wave amplitude (Mmax) obtained after electrical stimulation of the ulnar nerve at the wrist. ${ }^{26}$

MEP recruitment was studied at rest. The stimulus intensities studied were $90,100,110,120,130,140,150$ and $160 \%$ of the RMT. Eight pulses were delivered for each stimulus intensity, with stimulus intensities administered randomly. We excluded the first MEP for each trial from the analysis, as it could have been 'contaminated' by some untoward activity (that is, reflex, startle, tension/anxiety).

The peak-to-peak amplitudes of the MEPs recorded for each stimulus intensity were averaged and expressed as \% Mmax.

To determine whether the MEP changes during TMS stimulation also had a spinal component, we also examined H-reflexes from FDI muscle, before and after the TMS experiments.

\section{Statistical analysis}

Statistics were carried out using the software environment R (R Core Team (2013)), including the package npmv, which is available at http://CRAN.Rproject.org/package $=$ npmv and is based on the nonparametric multivariate inference methodology described by Bathke et al. ${ }^{27}$ The method is revolutionary because it allows to perform multiple analysis of variance-like nonparametric tests in situations, where the classical, parametric multiple analysis of variance is not applicable (for example, small sample size, no normal distribution).

We included MEP amplitudes (\% Mmax) for each stimulus intensity from 100 to $160 \%$ of the RMT in our analysis. As the covariance matrix of the ranked recruitment-curve measurements was singular, a nonparametric analysis of variance-type test was calculated. We included group (patients vs controls) as a between-subject factor and as within-factors (repeated measures; response variables), the MEP amplitudes for each stimulus intensity from 100 to $160 \%$ of RMT. The repeated measures design in this test treats each measure independently from its position in the sequence, thus accounting for the randomized delivery of the stimuli. Together with the test, we also provide relative effects. The relative effects can be understood as tendencies expressed as estimated probabilities.

The same nonparametric analysis of variance-type test, which was applied to all stimulus intensities at once, was also applied repeatedly as a univariate post hoc test to compare MEP amplitudes between patients and controls at single stimulus intensities from 100 to $160 \%$ of the RMT. The resulting $P$-values were then corrected for multiple comparisons according to Holm. These post hoc tests allowed us to determine at which intensities the values of the two groups differed significantly. To compare the MEP/Mmax amplitude ratio and RMT between groups, we applied the nonparametric analysis of variance-type test with group as between-subject factor and MEP/Mmax amplitude ratio and RMT as response variables, thus testing for group effects in these two measures. To test whether Hmax/Mmax ratio differed pre-to-post TMS within groups and between groups, we calculated a nonparametric $2 \times 2$ test with the R-package nparLD (Noguchi et al. 2012; http://cran.r-project.org/web/ packages/nparlLD). 
We report the test statistic (F-values), degrees of freedom and the $P$-values for global test results and for each significant post hoc test.

\section{RESULTS}

The analysis of the recruitment curve at different intensities yielded an analysis of variance-type test value of $\mathrm{F}_{(3.8,44.6)}=14.82$ with $P<0.001$, showing a significant main effect of group.

The relative effects given in Table 2 show that at each intensity of $110,130,140,150$ or $160 \%$, the probability that a randomly chosen patient exhibited larger response values compared with a randomly chosen participant from the full trial was $94-98 \%$. Post hoc tests revealed that groups differed significantly at intensities $110 \%$ (F $(1,11.7)=37.12 ; P<0.001), 130 \% \quad\left(\mathrm{~F}_{(1,11.7)}=21.13 ; P=0.003\right), 140 \%$ $\left(\mathrm{F}_{(1,11.7)}=32.75 ; P<0.001\right), 150 \%\left(\mathrm{~F}_{1,11.7)}=32.15 ; P<0.001\right)$ and $160 \%\left(\mathrm{~F}_{(1,11.7)}=35.71 ; P<0.001\right)$. The MEP recruitment curves of the two groups are shown in Figure 1. Comparing MEP/Mmax amplitude ratio and RMT between groups yielded no significant difference between groups $\left(\mathrm{F}_{(1.95,22.9)}=0.35 ; P=0.71\right.$; permutation test with

Table 2 Relative effects of the two groups at the different thresholds

\begin{tabular}{lccccccc}
\hline & \multicolumn{7}{c}{ Percent of stimulation } \\
\cline { 2 - 7 } Group & 100 & 110 & 120 & 130 & 140 & 150 & 160 \\
Controls & 0.36 & 0.02 & 0.18 & 0.06 & 0.03 & 0.03 & 0.02 \\
Patients & 0.63 & 0.98 & 0.82 & 0.94 & 0.97 & 0.97 & 0.98 \\
\hline
\end{tabular}

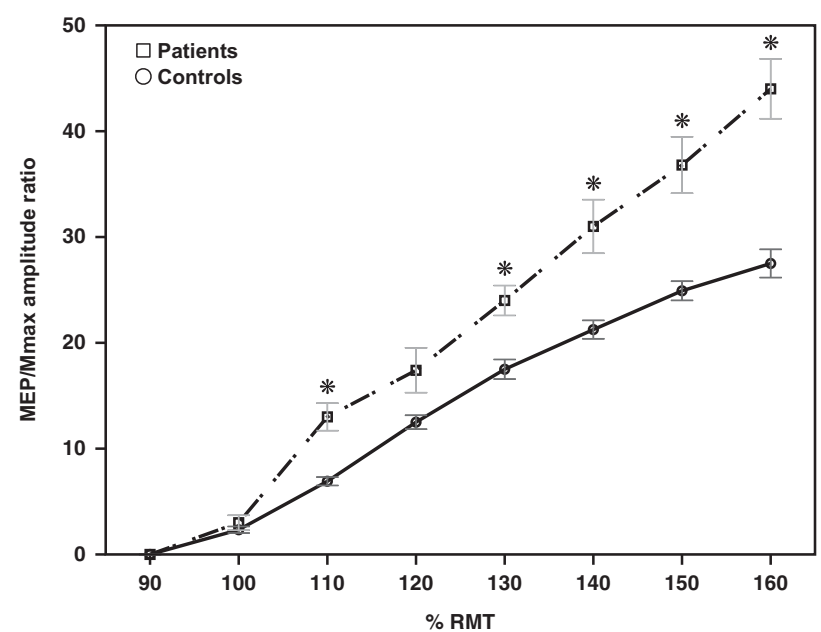

Figure $1 \mathrm{MEP}$ recruitment curve shows statistically significant increased values at 110,130,140, 150 and $160 \%$ of resting motor threshold (RMT) in the patients with spinal cord injury.

Table 3 Descriptive statistics

\begin{tabular}{lrrrrrrr}
\hline & \multicolumn{2}{c}{ Patients } & & \multicolumn{2}{c}{ Controls } & \multirow{2}{*}{ Significance } \\
\cline { 2 - 3 } & Mean & s.d. & & Mean & s.d. & \\
\hline RMT & 49.2 & 9.42 & 45.92 & 6.88 & - \\
Hmax/Mmax ratio before TMS & 0.59 & 0.10 & 0.38 & 0.14 & $*$ \\
Hmax/Mmax ratio after TMS & 0.59 & 0.10 & 0.57 & 0.13 & $*$ \\
\hline
\end{tabular}

Abbreviations: Hmax, maximal H-reflex amplitude; Mmax, maximal M-wave amplitude; RMT, resting motor threshold, expressed as \% of maximal stimulator output; SD, standard deviation. *Significant difference.
1000 permutations $P$-value $=0.74)$. The $2 \times 2$ test revealed that $\mathrm{Hmax} /$ Mmax ratio differed significantly between groups $\left(\mathrm{F}_{(1)}=4.91\right.$; $P=0.03)$ but not pre-to-post TMS $\left(\mathrm{F}_{(1)}=2.17 ; P=0.14\right)$, and there was no significant interaction between group and pre-to-post TMS $\left(\mathrm{F}_{(1)}=1.19 ; P=0.28\right)$. Both values were significantly higher in patients compared with healthy controls.

The means and s.d. of the other electrophysiological parameters (RMT, Hmax/Mmax ratio before and after TMS) are shown in Table 3.

\section{DISCUSSION}

The main finding of this study was that the growth of MEP amplitude, as a function of stimulus intensity-the recruitment curve, was significantly increased in SCI patients, whereas the cortical RMT of the FDI remained unchanged.

Different physiological processes and neuronal populations are likely to represent the physiological substrate of the stimulus/response curve. At low TMS intensity, the corticospinal volley often consists of a single I1-wave, whereas at higher stimulus intensities, the corticospinal volley resulting in the MEP becomes more complex because of the additional recruitment of later indirect waves (I2-I4 waves). ${ }^{28}$ These late I-waves are thought to depend on trans-synaptic activation of corticospinal axons through excitatory interneurons, which are controlled by several neurotransmitters/neuromodulators. ${ }^{29}$ Notably, the GABA-agonist lorazepam intake suppresses the amplitude of the later I-waves and the size of the MEP produced by high-intensity TMS, whereas the early I-wave and RMT remain unaffected. ${ }^{30}$

The amplitude of the MEP reflects the integrity of the corticospinal tract and the excitability of motor cortex and spinal level, as well as the conduction along the peripheral motor pathway to the muscles. ${ }^{3,31}$ As TMS activates cells with monosynaptic and polysynaptic connections with spinal motoneurons, MEP amplitude is sensitive to the excitability state of segmental neural circuits. ${ }^{32-35}$ By contrast, RMT provides information about a central core of neurons in the muscle representation in the motor cortex and is likely to reflect both neuronal membrane excitability, as well as non- $N$-methyl-D-aspartate receptor-mediated glutamatergic neurotransmission. ${ }^{29}$

Therefore, the electrophysiological pattern (altered MEP recruitment curve and normal RMT) that we found in SCI patients seems to be due to an enhanced spinal excitability rather than to an increased cortical excitability.

On the other hand, stimulus-response curve is thought to detect changes in cortical motor maps and to provide data equivalent to that provided by cortical maps. Although the present study did not include a formal MEP cortical mapping procedure, it has been demonstrated that increases in the cortical map area, for example, following ischemic anesthesia, are accompanied by concomitant increases in the slope of the corticospinal stimulus/response curve. ${ }^{36}$

The results of our study may thus also suggest that the cortical map for the FDI expands in patients with incomplete cervical SCI. This finding is consistent with the evidence from animal and human studies, demonstrating neuroplastic changes in sensorimotor cortex following changes of somatic afferents of motor output. ${ }^{37}$

Unmasking of preexisting synaptic connections, owing to disinhibition at cortical or subcortical levels, can be considered the mechanism underlying acute modulation of motor outputs. Although at the level of SCI lesion, a regenerative sprouting of interneurons may occur, above the lesion descending corticospinal axons extend new collaterals, and in the motor cortex new neurons are recruited along with the redistribution of projection neurons. The activation of functionally intact corticospinal neurons may depolarize a larger fraction of the 
spinal motoneuronal pool, thus shortening the rise time of the MEP recruitment curves.

Interestingly, our results are similar to those observed in conditions characterized by a more extreme sensorimotor deafferentation, such as ischemic nerve block, ${ }^{38}$ amputations ${ }^{39}$ and limb immobilization. ${ }^{40}$

A recent study reported significantly increased active motor threshold in patients with incomplete cervical SCI. ${ }^{18}$ This result was explained by the low density of motoneurons that innervate the hand muscles and seems to be in contrast with our findings. However, active motor threshold differs from RMT in that excitability of motoneurons in the spinal cord is enhanced by voluntary contraction of the target muscle, thus providing a measure of corticospinal excitability with greater dependence on the spinal segmental level excitability. ${ }^{28}$

There are several limitations of this preliminary study that must be recognized. The number of subjects tested is small. Our results apply to a small subgroup of patients with good motor recovery after incomplete cervical cord lesions. The situation might differ in patients with more severe lesions and/or functional deficits. Further studies in a large cohort of subjects with a more heterogeneous degree of lesion level and severity, as well as different time since injury, are warranted. Future investigations could provide further insights into these reorganizational changes, assess their relation with clinical changes and determine whether the observed abnormalities may serve as objective outcome measure in the design of clinical trials.

Another potential limitation is that the patients were taking baclofen, which has not been discontinued to avoid clinical worsening. Baclofen is known to act at the spinal, brainstem and cortical level. ${ }^{41-43}$ Nevertheless, all the selected patients were taking a similar dose of baclofen, ranging from 25 to $30 \mathrm{mg} /$ day. Thus, the cortical and spinal excitability are likely to be influenced in a similar manner in all subjects by the chronic intake of the antispastic drug.

To best of our knowledge, only one study has investigated the effects of baclofen on MEP recruitment curve after baclofen administration. ${ }^{44}$ In contrast to our results, baclofen was found to cause a reduction in the MEPs size. It should, however, be considered that findings obtained after a single administration may apply only to acute effects of the drug in healthy subject. Effects may be different if the drug is administered chronically, or if it is taken by patients with chronic SCI and successive brain reorganization, as in our study.

Anyway, our preliminary findings suggest that the reorganization of the corticospinal system might increase motor output and improve voluntary movement of the paretic hand. These mechanisms may be relevant for reorganizational processes and functional recovery after SCI. The data of our study thus provide insights into brain function changes due to neuroplasticity after SCI at the cortical and spinal level. Better understanding of cortical plasticity mechanisms in functional recovery may support the development of effective repair strategies in subjects with SCI.

\section{CONFLICT OF INTEREST}

The authors declare no conflict of interest.

1 Nardone R, Höller Y, Brigo F, Seidl M, Christova M, Bergmann J et al. Functional brain reorganization after spinal cord injury: systematic review of animal and human studies. Brain Res 2013; 1504: 58-73.

2 Moxon KA, Oliviero A, Aguilar J, Foffani G. Cortical reoprganization after spinal cord injury. Neuroscience 2014; 283c: 78-94.

3 Hallett M. Transcranial magnetic stimulation and the human brain. Nature 2000; 406 : 147-150.
4 Davey NJ, Smith HC, Wells E, Maskill DW, Savic G, Ellaway PH et al. Responses of thenar muscles to transcranial magnetic stimulation of the motor cortex in patients with incomplete spinal cord injury. J Neurol Neurosurg Psychiatry 1998; 65: 80-87.

5 Shimizu T, Hino T, Komori T, Hiraim S. Loss of the muscle silent period evoked by transcranial magnetic stimulation of the motor cortex in patients with cervical cord lesions. Neurosci Lett 2000; 286: 199-202.

6 Smith HC, Davey NJ, Savic G, Maskill DW, Ellaway PH, Jamous MA et al. Modulation of single motor unit discharges using magnetic stimulation of the motor cortex in incomplete spinal cord injury. J Neurol Neurosurg Psychiatry 2000a; 68: 516-520.

7 Smith HC, Savic G, Frankel HL, Ellaway PH, Maskill DW, Jamous MA et al. Corticospinal function studied over time following incomplete spinal cord injury. Spinal Cord 2000b; 38: 292-300.

8 Saturno E, Bonato C, Miniussi C, Di Lazzaro V, Callea L. Motor cortex changes in spinal cord injury: a TMS study. Neurol Res 2008; 30: 1084-1085.

9 Butler JE, Larsen TS, Gandevia SC, Petersen NT. The nature of corticospinal paths driving human motoneurones during voluntary contractions. J Physiol 2007; 584: $651-665$.

10 Roy FD, Zewdie ET, Gorassini MA. Short-interval intracortical inhibition with incomplete spinal cord injury. Clin Neurophysiol 2011; 122: 1387-1395.

11 Kujirai T, Caramia MD, Rothwell JC, Day BL, Thompson PD, Ferbert A et al. Corticocortical inhibition in human motor cortex. J Physiol 1993; 471: 501-519.

12 Ilic' TV, Meintzschel F, Cleff U, Ruge D, Kessler KR, Ziemann U. Short-interval pairedpulse inhibition and facilitation of human motor cortex: the dimension of stimulus intensity. J Physiol 2002; 545: 153-167.

13 Wagle-Shukla A, Ni Z, Gunraj CA, Bahl N, Chen R. Effects of short interval intracortical inhibition and intracortical facilitation on short interval intracortical facilitation in human primary motor cortex. J Physiol 2009; 587: 5665-5678.

14 Garry MI, Thomson RH. The effect of test TMS intensity on short-interval intracortical inhibition in different excitability states. Exp Brain Res 2009; 193: 267-274.

15 Ortu E, Deriu F, Suppa A, Tolu E, Rothwell JC. Effects of volitional contraction onintracortical inhibition and facilitation in the human motor cortex. J Physiol 2008; 586: 5147-5159.

16 Peurala SH, Muller-Dahlhaus JF, Arai N, Ziemann U. Interference of short-interval intracortical inhibition (SICI) and short-interval intracortical facilitation (SICF). Clin Neurophysiol 2008; 119: 2291-2297.

17 Lotze M, Laubis-Herrmann U, Topka H. Combination of TMS and fMRI reveals a specific pattern of reorganization in $\mathrm{M} 1$ in patients after complete spinal cord injury. Restor Neurol Neurosci 2006; 24: 97-107.

18 Freund P, Rothwell J, Craggs M, Thompson AJ, Bestmann S. Corticomotor representation to a human forearm muscle changes following cervical spinal cord injury. Eur $J$ Neurosci 2001; 34: 1839-1846.

19 Inghilleri M, Berardelli A, Cruccu G, Manfredi M. Silent period evoked by transcranial stimulation of the human cortex and cervicomedullary junction. J Physiol 1993; 466: 521-534.

20 Hess CW, Mills KR, Murray NM. Responses in small hand muscles from magnetic simulation of the human brain. J Physiol 1987; 388: 397-419.

21 Devanne H, Lavoie BA, Capaday C. Input-output properties and gain changes in the human corticospinal pathway. Exp Brain Res 1997; 114: 329-338.

22 Kimiskidis VK, Papagiannopoulos S, Sotirakoglou K, Kazis DA, Kazis A, Mills KR. Silent period to transcanial magnetic stimulation: construction and properties of stimulusresponse curves in healthy volunteers. Exp Brain Res 2005; 163: 21-31.

23 Boroojerdi B, Battaglia F, Muellbacher W, Cohen LG. Mechanisms influencing stimulus-response properties of the human corticospinal system. Mechanisms of deafferentation-induced plasticity in human motor cortex. Clin Neurophysiol 2001; 112: 931-937.

24 Marino RJ, Barros T, Biering-Sorensen F, Burns SP, Donovan WH, Graves DE et al. ASIA Neurological Standards Committee 2002. International standards for neurological calssification of spinal cord injury. J Spinal Cord Med 2003; 26: S50-S56.

25 Rossini PM, Barker AT, Berardelli A, Caramia MD, Caruso G, Cracco RQ et al. Non invasive electrical and magnetic stimulation of the brain, spinal cord and roots: basic principles and procedures for routine clinical application: report of IFCN committee. Electroencephalogr Clin Neurophysiol 1994; 91: 79-92.

26 Murray MMF. Motor evoked potentials. In: Aminoff M (ed). The Textbook of Electrodiagnosis in Clinical Neurology, 4th edn. 1999, pp 549-568.

27 Bathke AC, Harrar SW, Madden LV. How to compare small multivariate samples using nonparametric tests. Comput Stat Data Anal 2008; 52: 4951-4965.

28 Di Lazzaro V, Ziemann U, Lemon RN. State of the art: physiology of transcranial motor cortex stimulation. Brain Stimul 2008; 1: 345-362.

29 Ziemann U. Pharmaco-transcranial magnetic stimulation studies of motor excitability. Handb Clin Neurol 2013; 116: 387-397.

30 Di Lazzaro V, Oliviero A, Meglio M, Cioni B, Tamburrini G, Tonali P et al. Direct demonstration of the effect of lorazepam on the excitability of the human motor cortex. Clin Neurophysiol 2000; 111: 794-799.

31 Kobayashi M, Pascual-Leone A. Transcranial magnetic stimulation in neurology. Lancet Neurol 2003; 2: 145-156.

32 Burke D, Hicks R, Gandevia SC, Stephen J, Woodforth I, Crawford M. Direct comparison of cortispinal volleys in human subjects to transcranial magnetic and electrical stimulation. J Physiol 1993; 470: 383-393.

33 Nielsen J, Petersen N. Changes in the effect of magnetic brain stimulation accompanying voluntary dynamic contraction in man. J Physiol 1995; 484: 777-789.

34 Schneider C, Lavoie BA, Barbeau H, Capaday C. Timing of cortical excitability chnages during time of movements superimposed on tonic motor activity. J Appl Physiol 2004; 97: 2220-2227. 
35 Geertsen SS, Zuur AT, Nielsen JB. Voluntary activation of ankle muscle is accompanied by subcortical facilitation of their antagonists. J Physiol 2010; 588: 2391-2402.

36 Ridding MC, Rothwell JC. Stimulus/response curves as a method of measuring motor cortical excitability in man. Electroencephalogr Clin Neurophysiol 1997; 105 340-344

37 Sanes JN, Donoghue JP. Plasticity and primary motor cortex. Annu Rev Neurosc 2000; 23: 393-415.

38 Ziemann U, Corwell B, Cohen LG. Modulation of plasticity in human motor cortex after forearm ischemic nerve block. J Neurosci 1998; 18: 1115-1123.

39 Chen R, Corwell B, Yaseen Z, Hallett M, Cohen LG. Mechanisms of cortical reorganization in lower-limb amputees. J Neurosci 1998; 18: 3443-3450.
40 Zanette G, Manganotti P, Fiaschi A, Tamburin S. Modulation of motor cortex excitability after upper limb immobilization. Clin Neurophysiol 2004; 115: 1264-1275.

41 Kofler M, Donovan WH, Loubser PG, Berić A. Effects of intrathecal baclofen on lumbosacral and cortical somatosensory evoked potentials. Neurology 1992; 42: 864-868.

42 Kumru H, Kofler M. Effect of spinal cord injury and of intrathecal baclofen on brainstem reflexes. Clin Neurophysiol 2012; 123: 45-53.

43 Stetkarova I, Kofler M. Differential effect of baclofen on cortical and spinal inhibitory circuits. Clin Neurophysiol 2013; 124: 339-345.

44 Willerslev-OIsen M, Lundbye-Jensen J, Petersen TH, Nielsen JB. The effect of baclofen and diazepam on motor skill acquisition in healthy subjects. Exp Brain Res 2011; 213: 465-474. 